\title{
Effects of Non-Local Thermodynamic Equilibrium (NLTE) on Molecular Opacities
}

\author{
Hollis R. Johnson
}

Astronomy Department SW 319, Indiana University, Bloomington, IN 47405, U.S.A.

\section{Introduction and Basic Concepts}

Molecules increase in importance in stellar atmospheres as the temperature falls along the spectral sequence for all luminosity classes. Among their many roles molecular features provide the basis for spectral classification (temperature and chemical composition) in $\mathrm{M}, \mathrm{S}$, and $\mathrm{C}$ stars; they constitute important opacity sources across the spectrum from the ultraviolet to the infrared; they influence the energy budget and hence the kinetic temperature in the outer photospheric layers; they may contribute significantly to the radiative levitation of the atmosphere; they form masers; they may cause or facilitate mass loss; and they are indicative of physical conditions and processes from the photosphere to the outer circumstellar envelope.

Most of the known molecular opacity is produced by only a few molecules $-\mathrm{H}_{2}{ }^{+}, \mathrm{H}_{2}{ }^{-}$, pressure-induced $\mathrm{H}_{2}$ opacity, and opacities of $\mathrm{CH}, \mathrm{NH}, \mathrm{MgH}, \mathrm{SiH}$, $\mathrm{CO}, \mathrm{SiO}, \mathrm{CN}, \mathrm{CS}$, and $\mathrm{SiS}$, of which $\mathrm{CN}$ is usually the most important. In normal oxygen-rich stars there are also $\mathrm{TiO}, \mathrm{VO}, \mathrm{H}_{2} \mathrm{O}$, and $\mathrm{OH}$, and in carbon-rich stars there are also $\mathrm{C}_{2}, \mathrm{HCN}, \mathrm{C}_{2} \mathrm{H}_{2}$, and $\mathrm{C}_{3}$. Finally, there are such hitherto neglected molecules as $\mathrm{ZrO}, \mathrm{FeH}, \mathrm{C}_{2} \mathrm{H}, \mathrm{AlOH}$, and perhaps several more. Despite the importance of molecular opacities, little attention has been given to the effects of NLTE, as is clear from recent reviews (see Tsuji 1986).

Competition between the processes of molecular formation and destruction determines the number density of each molecular species and each energy level. If these processes occur at any but their equilibrium rates, they will lead to overpopulation or underpopulation of either molecular states or the density of the molecules themselves. In chromospheres, shocks, winds, molecular-cluster and dust-formation regions, and maser-formation regions, we expect major departures from LTE. However, our focus here is on the photosphere.

In strict thermodynamic equilibrium (TE) each process is exactly balanced by its inverse process at every velocity and frequency, and the distribution functions of equilibrium statistical mechanics hold. Such an ideal regime is approached deep inside a star. However, TE is not expected to hold exactly in the presence of temperature gradients or anisotropies in the radiation field, both of which 
must occur in stellar atmospheres. Although strict TE cannot be expected to hold, astrophysicists have long employed a slightly relaxed form of TE - local thermodynamic equilibrium (LTE). In this, one still expects (a) particle velocities are Maxwellian because of the short mean free path and high elastic collision frequency; (b) electrons are distributed among atomic and molecular states according to the Boltzmann law; (c) atoms are distributed among stages of ionization according to the Saha law; and (d) molecular dissociative equilibrium (sometimes called chemical equilibrium) holds, but (e) the radiation field might not be planckian because of the long mean free path of the photons and the necessary anisotropy of the radiation field near the stellar surface.

In fact, the specific intensity of radiation, $\mathrm{I}_{\nu}$, is described by

$$
\mu \frac{d I_{\nu}}{d r}=\xi_{\nu}-\chi_{\nu} I_{\nu}
$$

where $\mathrm{I}_{\nu}$ is the specific intensity $\left[\mathrm{erg} \mathrm{cm} \mathrm{cm}^{-2} \mathrm{~s}^{-1} \mathrm{~Hz}^{-1} \mathrm{sr}^{-1}\right.$, for example], $\mathrm{r}$ is an outwardly directed distance, $\chi_{\nu}$ is the linear extinction coefficient $\left[\mathrm{cm}^{-1}\right], \xi_{\nu}$ is the volume emission coefficient $\left[\mathrm{erg} \mathrm{cm}^{-3} \mathrm{~s}^{-1} \mathrm{sr}^{-1} \mathrm{~Hz}^{-1}\right]$, and $\mu=\cos \theta$, where $\theta$ is the angle between the normal to the infinitesmal surface and the direction of the pencil of radiation. If we define the optical depth, $\mathrm{d} \tau_{\nu}=-\chi_{\nu} \mathrm{dr}$, and the source function, $\mathrm{S}_{\nu}=\xi_{\nu} / \chi_{\nu}$, we obtain the familiar form (see also Scholz \& Wehrse this volume) of the equation of radiative transfer

$$
\mu \frac{d I_{\nu}}{d \tau_{\nu}}=I_{\nu}-S_{\nu} .
$$

In $\mathrm{TE}, \mathrm{I}_{\nu}=\mathrm{B}_{\nu}(\mathrm{T})$, the Planck function. However, in a stellar atmosphere, this is not generally true. The assumption that all the other distribution functions (a) - (d) above are never theless valid leads to the condition of "local" thermodynamic equilibrium (LTE), and this is a "standard" assumption in classical stellar atmospheres. The reader will surely notice the inconsistency of the LTE idea that the radiation field might depart from its LTE value without influencing the distributions of electrons, molecules or atoms. This logical inconsistency is a small price to pay, however, for the convenience of using the Boltzmann and Saha equations to calculate number densities. The question is not: Is LTE valid? Of course it is not strictly valid in stellar atmospheres because of the inconsistency noted. The question is: How bad are the LTE results?

A broad view of NLTE, including all departures from the idealized situation described above, is illustrated in Fig. 1. which shows a stylized representation of the formation and destruction of a diatomic molecule $\mathrm{AB}$ from its constituent neutral atoms $\mathrm{A}$ and $\mathrm{B}$, the populations of each of which might be influenced by interactions with other molecules or other stages of ionization. Molecules might also be formed by any of several other processes, such as exchange reactions.

NLTE effects can enter in many places, as shown in Fig. 2. Populations of levels, and hence source functions and optical depths, depend on the competing rates of production and destruction. If there is a steady state, often called statistical equilibrium, then, for the number density of the particles corresponding 
to any state $\mathrm{j}$ (which could be an atomic or molecular energy level), $\mathrm{dn}_{j} / \mathrm{dt}=$ (production rate of $n_{j}$ ) - (destruction rate of $n_{j}$ ) $=0$. That is, the sum of all processes leading to the creation of a particle in state $\mathrm{j}$ is equal to the sum of all processes taking particles out of state $\mathrm{j}$ (see Mihalas 1978; Anderson 1989).

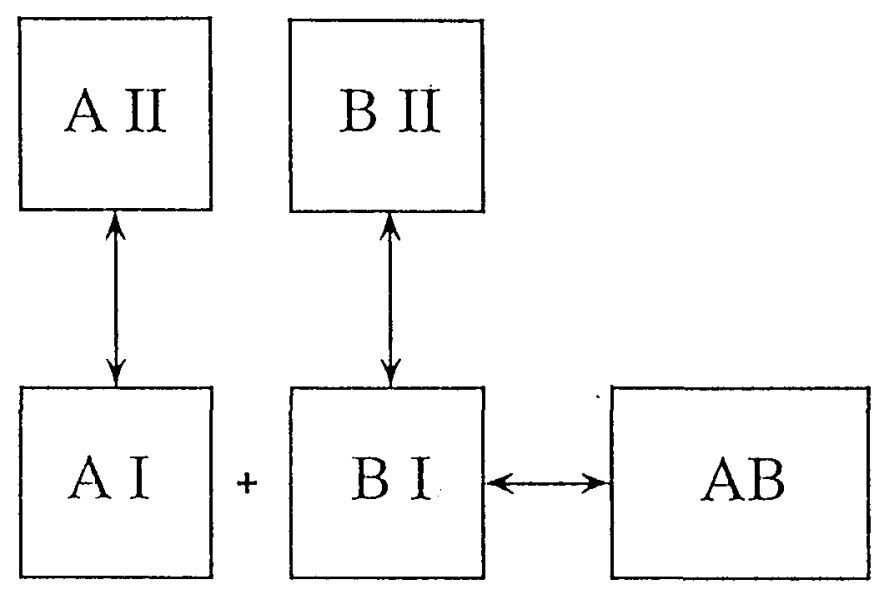

Fig. 1. Schematic representation of the formation of a diatomic molecule AB from neutral atoms $A$ and $B$.

Each process involves a forward and a reverse rate or process. Considering only states $\mathrm{i}$ and $\mathrm{j}$, if $\mathrm{P}$ stands for the rate of any process, we can describe the steady-state equilibrium between $i$ and $j$ by

$$
n_{i} P_{i j}=n_{j} P_{j i}
$$

where $\mathrm{n}_{i}$ is the number density of particles of species $\mathrm{i}$, and $\mathrm{P}_{i j}$ is the rate per particle of any process transferring particles from one state $i$ to another state $j$. Normally, many states will be connected to the $j$ 'th state, and there will be many terms in the equation. The ratio of the number densities of $j$ and $i$ in the simple case where no other interactions occur is given by

$$
\frac{n_{j}}{n_{i}}=\frac{P_{i j}}{P_{j i}} .
$$

In TE the rates $\mathrm{P}_{i j}$ and $\mathrm{P}_{j i}$ will balance in such a way that their ratio is just the relevant equilibrium distribution function. More generally, for an energy level $\mathrm{j}$ in a system of $\mathrm{N}$ levels,

$$
n_{j} \Sigma_{i} P_{j i}+n_{j} P_{j K}=\Sigma_{i} n_{i} P_{i j}+n_{K} P_{K j} ; j=1,2 \ldots N,
$$

where $\mathrm{K}$ represents the continuum or the dissociated state. 


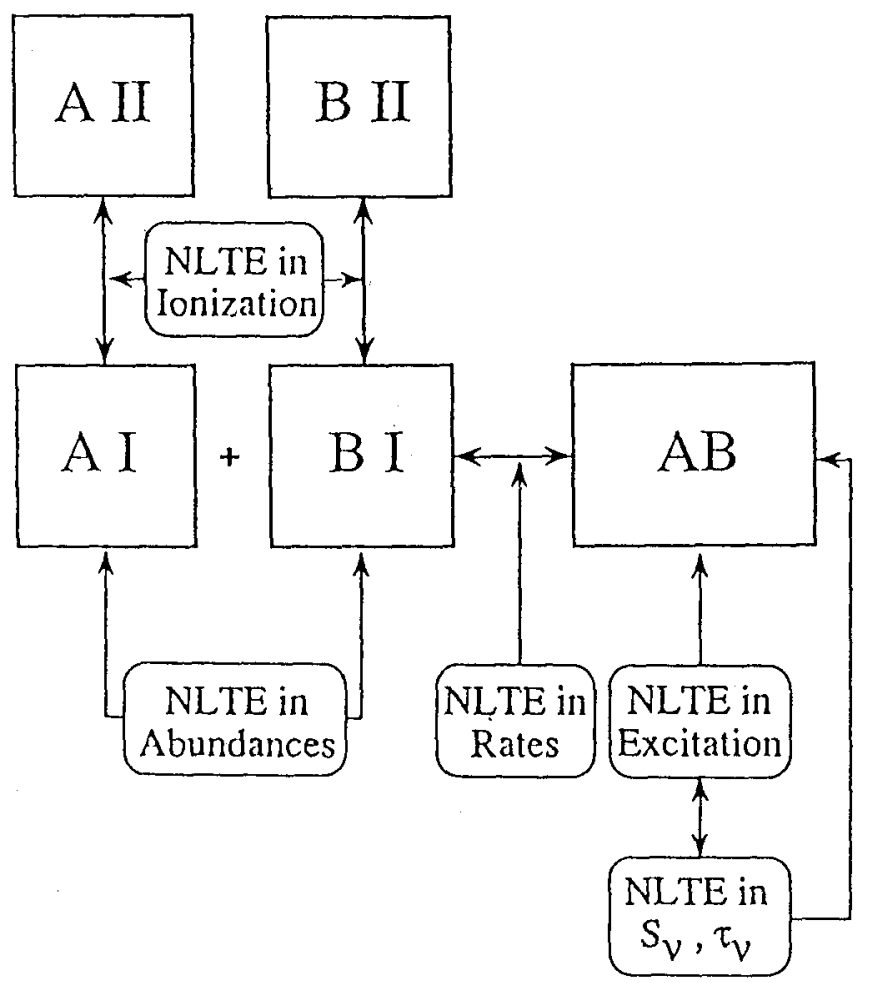

Fig. 2. Schematic representation of the points (formation and destruction rates, excitation mechanism, spectral lines, and the pool of constituent atoms) at which departures from LTE (NLTE) in the rates might occur for the simple situation shown in Fig. 1. Any NLTE effect might influence the populations of the molecular energy levels and the opacity.

In general, each transition can be made radiatively or collisionally, so that

$$
P_{i j}=R_{i j}+C_{i j}
$$

where $\mathrm{R}$ and $\mathrm{C}$ are the radiative and collisional rates. Since the colliding particles have a maxwellian velocity distribution, we can compute the collisional rate, $\mathrm{C}$, as an integral over velocity and cross section

$$
C_{i j}=C_{i j}(Q, n, T)
$$

where $Q$ represents a cross section. In steady-state equilibrium, all the relevant cross sections must be known, and astrophysicists depend on quantum chemists and laboratory spectroscopists for these values. Note that the density and temperature of the colliding species (usually electrons, $\mathrm{H}$ or $\mathrm{He}$ atoms, or $\mathbf{H}_{2}$ molecules) are local quantities which furthermore are in equilibrium, so that 
if one computes

$$
\frac{n_{j}}{n_{i}}=\frac{C_{i j}}{C_{j i}}
$$

the result will be the TE value.

For the radiative rates one has

$$
R_{i j}=R_{i j}\left(f, I_{\nu}\right)
$$

where $f$ is an oscillator strength or absorption cross section and, as repeatedly stressed at throughtout this book, these must be accurately known. If $I_{\nu}=$ $\mathrm{B}_{\nu}(\mathrm{T})$, the ratio of $\mathrm{n}_{j} / \mathrm{n}_{i}$ would again be the TE value. However, in general this is not true, for $\mathrm{I}_{\nu}\left(\tau_{\nu}\right)$ given by Eq. (2).

Molecular lines are often assumed to be formed by pure absorption, in which case the source function becomes the Planck function

$$
S_{\nu}^{l}\left(\tau_{\nu}\right)=B_{\nu}(T)
$$

Even under these circumstances, however, $I_{\nu}$ is not Planckian! A more general approximation to the line source function is the frequency-independent form

$$
S_{\nu}^{l}=\frac{2 h \nu^{3}}{c^{2}} \frac{1}{\frac{n_{i} g_{j}}{n_{j} g_{i}}-1},
$$

where $\mathrm{j}$ is the upper and $\mathrm{i}$ the lower state and $\mathrm{g}$ is the statistical weight.

Substitution of Eq. (5) into Eq. (12) leads to a form which is a linear combination of pure absorption and complete redistribution (pure non-coherent scattering):

$$
S_{\nu}^{l}=\epsilon B_{\nu}(T)+(1-\epsilon) \bar{J}
$$

where

$$
\bar{J}=\int_{0}^{\infty} J_{\nu} \phi_{\nu} d \nu,
$$

$\phi_{\nu}$ is the normalized absorption coefficient, and $\epsilon$ is related to the fraction of photons re-emitted thermally.

To obtain a physical feel for the radiation field, one might imagine that $I_{\nu}$ or its angle average, $\mathrm{J}_{\nu}$, can be parameterized as

$$
J_{\nu}=W B_{\nu}\left(T_{r}\right)
$$

where $\mathrm{W}$ is a dilution factor (the fractional solid angle filled by incoming radiation at the point of interest) and $T_{r}$ is the radiation temperature. Thus $\mathrm{J}_{\nu}$ might differ from $\mathrm{B}_{\nu}(\mathrm{T})$ either because of the dilution of the radiation field near the surface $(\mathrm{W}<1)$ or because the (wavelength-dependent!) "radiation temperature" $\left(\mathrm{T}_{r}\right)$ departs from the local electron temperature $(\mathrm{T})$.

The complexity of the true (NLTE) situation is now apparent. The number densities of the relevant atomic and molecular states and species depend upon the radiative and collisional rates, and the radiative rates depend on the radiation field (Eq. 9). The radiation field $\left(I_{\nu}\right)$ depends upon the source functions and 
optical depths (Eq. 2), which depend upon the number densities of relevant states (Eqs. 1 and 11). Often the coupling between the radiation field and the energy levels is very strong, and special mathematical techniques are needed to solve the system of equations.

As is clear from the previous (Eqs. 5, 9, and 14), departures from LTE in level populations will occur if (a) radiative rates exceed collisional rates and (b) the radiation field is non-planckian $\left(I_{\nu} \neq B_{\nu}(T)\right)$. Stated differently, level populations will be driven to their LTE values if collisional rates (which occur at their equilibrium values) dominate radiative rates or if radiative rates proceed at their equilibrium value (the intensity is the Planck function). Deep in the photosphere particle densities are high $\left(\sim 10^{15}-10^{18} \mathrm{~cm}^{-3}\right)$, and collisional rates are high. Furthermore, optical depths are large, and the radiation field approaches a planckian radiation field. Under these conditions, we expect LTE to be an excellent approximation. In the outer photosphere particle densities are lower $\left(10^{11}-10^{14} \mathrm{~cm}^{-3}\right)$, and electron densities are far lower, and departures from a plankian radiation field arise both from the existence of the surface $(\tau=$ 0 ) with its consequent anisotropy in the radiation field and from the frequency dependence of the absorption coefficients and therefore of the surface flux. Effects of NLTE are to be expected. In chromospheres, coronae, winds, and CSE's, NLTE effects dominate, and the full coupled radiative-transfer and steady-state equations (or even non-static equations) must be employed.

Two diametrically opposite philosophical approaches to NLTE in stellar photospheres have therefore arisen. One position, the one almost always taken in calculations of molecular opacities, is that the assumption of LTE with its attendant simplicity should be made until clearly discrepant results force us to the tedious NLTE calculations. The other position is to begin NLTE calculations at the outset. There is hardly any middle ground.

For which molecular levels and transitions are NLTE effects likely to be important? Molecular energy states are classified as rotational, vibrational, and electronic. Electronic levels and transitions involve energies of a few $(2-6) \mathrm{eV}$, whereas the average kinetic energy per particle is of the order of $0.3 \mathrm{eV}$. Furthermore, the agents of electronic collisions in molecules, as in atoms, are electrons, the density of which decreases rapidly toward lower temperatures as abundant elements $(\mathrm{H}, \mathrm{C}, \mathrm{N}, \mathrm{O})$ become neutral. These transitions are therefore quite susceptible to NLTE effects (Thompson 1973; Mount et al. 1975; Hinkle \& Lambert 1975; Anderson 1989; Ayres \& Wiedemann 1989). Fortunately, many electronic molecular transitions fall in the violet and ultraviolet spectral regions where, due to the low stellar flux, their effects on the overall opacity and therefore on the atmospheric structure is minor. By contrast, rovibrational transitions have energies of the order of $0.5 \mathrm{eV}$, and rotational transitions have energies of the order of $0.05 \mathrm{eV}$. Since these are close to the average energy per particle in cool stellar atmospheres, many particles are available for collisions. Furthermore, these latter collisions are more geometric than coulombic and are mediated by neutral particles, especially neutral hydrogen atoms, which are relatively abundant even in the low-density atmospheres of red-giant stars. 
Direct measurements from spacecraft show that both the absolute stellar ultraviolet flux and the fractional ultraviolet flux in molecule-rich giants are weaker than in warmer stars but still quite significant (Steiman-Cameron et al. 1985; Schrijver 1987; Judge 1989). In fact, radiation temperatures $\left(\mathrm{T}_{r}\right)$ at violet and ultraviolet wavelengths may easily exceed the electron temperature in the outer photosphere and temperature-minimum region, especially if chromospheric photons are also included (Luttermoser 1993). For example, the color (radiation) temperature in $\alpha$ Ori never falls below $2950 \mathrm{~K}$ at any wavelength, and it rises sharply at wavelengths shortward of $300 \mathrm{~nm}$ to values of $3,000-4,000$ $\mathrm{K}$ (Carpenter et al. 1993). By contrast, the temperature minimum must have an electron temperature near $2400 \mathrm{~K}$. The ultraviolet radiation field is relatively much stronger in cool dwarfs. It is this relatively strong ultraviolet radiation field that causes the overionization and overexcitation in both molecules and atoms found in the outer photosphere in several studies (see individual molecules).

We now summerize research on a few specific molecules.

\section{$2 \mathrm{H}_{2}$}

Because it is the most abundant element, the equilibrium of hydrogen is fundamental to all hydrogen-containing molecules and their opacities. Although it has no bands of significance except through pressure-induced opacity (Borysow this volume), the molecule $\mathrm{H}_{2}$ has an important indirect influence on the opacity through its influence on the abundance of $\mathrm{H}^{-}$, whose opacity is important or dominant in all molecule-producing atmospheres except H-deficient objects.

A pioneering study of the actual (NLTE) equilibrium for both $\mathrm{H}_{2}$ and $\mathrm{H}^{-}$was reported in a classic paper by Lambert \& Pagel (1968), who set up and solved a small network of equations governing the equilibrium of $\mathrm{H}^{-}$. From considerations of several mechanisms of formation and destruction, they found the most important destruction mechanisms for $\mathrm{H}^{-}$in stars of spectral type $\mathrm{F} 0-\mathrm{K} 0$ to be photodetachment $\left(\mathrm{H}^{-}+\mathrm{h} \nu \rightarrow \mathrm{H}+\mathrm{e}\right)$ and associative detachment by neutral hydrogen atoms $\left(\mathrm{H}^{-}+\mathrm{H} \rightarrow \mathrm{H}_{2}+\mathrm{e}\right)$. Rates then available led to their conclusion that "NLTE effects are probably absent." Because of its indirect influence on the $\mathrm{H}^{-}$abundance, the formation of $\mathrm{H}_{2}$, for which 3-body recombinations $(3 \mathrm{H} \rightarrow$ $\mathrm{H}_{2}+\mathrm{H}$ ) seemed to dominate, was also examined. Relative to the reservoir of neutral hydrogen the number density of $\mathrm{H}_{2}$ was also found to be in TE. A larger network of equations governing the equilibrium of $\mathrm{H}^{-}, \mathrm{H}, \mathrm{H}^{+}, \mathrm{H}_{2}$, and $\mathrm{H}_{2}{ }^{+}$in a form suitable for calculation by computers was set up, but not solved, by Lites \& Mihalas (1984). Most modern calculations of $\mathrm{H}^{-}$and $\mathrm{H}_{2}$ have cited this early research and have either assumed $\mathrm{H}^{-}$to be in LTE relative to $\mathrm{H}$ (Vernazza et al. 1981) or have calculated it to be so from a simplified set of equations (Anderson 1989). In both these latter calculations $\mathrm{H}_{2}$ was assumed to be in TE relative to $\mathrm{H} \mathrm{I}$ and it was analytically eliminated from the $\mathrm{H}^{-}$network of equations.

As a check on the abundance of $\mathrm{H}_{2}$, and therefore on dissociative equilibrium calculations, observations of the infrared quadrupole transitions of $\mathrm{H}_{2}$ are of paramount importance, and a fair sample of these are newly available (Hinkle et 
al. 1993), including observations of about $30 \mathrm{M}, \mathrm{S}$, and C stars of all variability classes and of the mira variable star $\chi$ Cyg throughout its cycle. A few $\mathrm{H}_{2}$ lines, especially the 1-0 S(0) and $\mathrm{S}(1)$ lines, were seen in most stars (the strongest lines being in S-type miras), and their strengths were roughly in agreement with the expectations of dissociative (chemical) equilibrium calculations. The great strengths of the stronger observed lines requires their formation over a large range of the stellar atmosphere, and, in extreme cases, up to $0.1 \mathrm{M}_{\odot}$ is contained in the atmosphere. Part of the line was found to originate in near-photospheric circumstellar material slightly shifted in velocity relative to the photosphere. It had earlier been suggested that if $\mathrm{H}_{2}$ is produced by three-body recombination, the recombination would be so slow in the low densities in mira atmospheres that $\mathrm{H}$ would remain neutral (not associated) between shocks (Bowen 1988). However, these observations disclose that, after the passage of a pulsationally driven shock through the atmosphere, the $\mathrm{H}_{2}$ lines reappear almost as quickly as do $\mathrm{CO}$ and $\mathrm{TiO}$ lines, forcing the conclusion that recombination proceeds more rapidly than expected, which in turn demands higher densities of $\mathrm{H} \mathrm{I}$ or consideration of processes other than three-body recombination. An increase in the rate of association of $\mathrm{H}$ into $\mathrm{H}_{2}$ would tend to drive the number density of $\mathrm{H}_{2}$ closer to its LTE value. However, in such a complex system as shocks in the pulsating atmosphere of a mira variable star, a completely self-consistent NLTE solution is required, and that is still in the future.

\section{$3 \mathrm{CH}$}

An early (perhaps the earliest) calculation of NLTE in CH (Hinkle \& Lambert 1975), noted that the three observed electronic transitions of $\mathrm{CH}: \mathrm{A}^{2} \Delta-\mathrm{X}^{2} \Pi$, $\mathrm{B}^{2} \Sigma^{-}-\mathrm{X}^{2} \Pi$, and $\mathrm{C}^{2} \Sigma^{+}-\mathrm{X}^{2} I I$ have very low electronic oscillator strengths, so that their contribution to the opacity in the Sun is small, while the stronger transitions lie so far to the ultraviolet $(137.0 \mathrm{~nm})$ that their contribution to the opacity is negligible. That conclusion is strengthened as one goes toward lower temperatures.

A detailed calculation of the $\mathrm{CH}(\mathrm{A}-\mathrm{X}), \mathrm{CH}(\mathrm{B}-\mathrm{X})$, and $\mathrm{CH}(\mathrm{C}-\mathrm{X})$ systems in the solar atmosphere by the methods outlined in Section 8 was made by Anderson (1989). Interesting departures from LTE were found in all three systems in the outer solar photosphere in the sense that higher-lying levels were overpopulated relative to lower-lying levels. The departures begin sharply above log $m=2.0$ in this radiative-equilibrium model, where $m$ is mass column density $\left(\mathrm{g} \mathrm{cm}^{-2}\right)$, just outside the temperature-minimum region in the real solar atmosphere. The physical reasons for the over excitation are related to the relatively strong ultraviolet radiation field as sketched in the last part of the Introduction.

A recent comparison of values for the carbon abundances in the solar atmo sphere (Grevesse et al. 1991) shows that an LTE abundance analysis of the CH (A-X) electronic system yields the same value for the carbon abundance as do the rotation-vibration bands in the ground state, and that value agrees closely with the mean value of other abundance indicators. This agreement is impor- 
tant, for it demonstrates that any NLTE effects are confined to the outermost layers where they may slightly affect the line cores but probably do not affect the opacity (which for $\mathrm{CH}$ in the sun is of minor importance anyway).

\section{$4 \mathrm{CN}$}

Bands of the red system of $\mathrm{CN}\left(\mathrm{A}^{2} \Pi-\mathrm{X}^{2} \Sigma^{+}\right)$are conspicuous in the red/nearIR spectral region $(0.7-1.5 \mu \mathrm{m})$ and provide important opacity in cool stars of all chemical composition, but we know of no evidence from either observation or theory for departures from the predictions of LTE in these lines.

Calculations of NLTE for the violet system of CN $\left(\mathrm{B}^{2} \Sigma^{+}-\mathrm{X}^{2} \Sigma^{+}\right)$have been carried out for the Sun (Mount \& Linsky 1975) and for Arcturus (Mount et al. 1975). Because of the obvious computational difficulties of such a complex system, the analysis was carried out only for rotational transitions from vibrational states $v^{\prime \prime}=0$ to $v^{\prime}=0$, which form the bandhead at $388.3 \mathrm{~nm}$. This amounted to 32 overlapping doublets corresponding to the P1 and P2 divisions of the P branch; the weaker $\mathrm{Q}$ branch was ignored. All rotational transitions in both the upper and lower electronic states were assumed to be in LTE. The equivalent-twolevel-atom formulation of the source function was adopted, the line absorption coefficient was taken to be doppler, and complete redistribution was assumed in the photon redistribution function. For the Sun, significant NLTE effects, as much as a factor of 2.0 in the ratio $S^{l} / B_{\nu}(T)$, were found for all four atmospheric models tested, including some with inhomogeneities. It was concluded that this factor could cause an increase of $0.2 \mathrm{dex}$ in the abundance of carbon. However, these workers obtained an abundance of $\mathrm{C}$ lower by $0.2-0.3$ dex than the value of 8.60 commonly accepted (Grevesse et al. 1991), and this unexplained difference has cast an uncertainty over their results.

Because of the low density in the atmosphere of the red giant Arcturus, values of $\epsilon$ were so low that most of the band head was formed by (pure noncoherent) scattering. Possible errors in the abundance of $\mathrm{C}$ and $\mathrm{N}$ resulting from the neglect of NLTE are again approximately $0.2 \mathrm{dex}$, which is of the same order as errors introduced by uncertainties in the absolute calibration of the spectra, the molecular oscillator strengths, and the turbulence. The authors warn against an extrapolation of these results to other molecules and other stars (Mount et al. 1975).

\section{$5 \mathrm{CO}$}

Exactly twenty years ago there appeared two calculations that set the stage for much of the later interesting work on CO: the tentative suggestion that $\mathrm{CO}$ might be an important coolant in stellar atmospheres of intermediate and low temperatures (Johnson 1973) and the pioneering calculations of NLTE in CO (Thompson 1973). Both of these intertwined effects have been treated in much more detail since then. Early calculations showed a very large cooling 
effect; subsequent calculations show a smaller but still important effect (Ayres \& Weidemann 1989; Mauas et al. 1990; Wiedemann \& Ayres 1991; Plez et al. 1992). CO is important to the energy budget in the outer layers where its strong lines are formed because most other lines have become optically thin. However, we concentrate on the NLTE aspects here, leaving the tantalizing questions of $\mathrm{CO}$ cooling and possible consequent bifurcation to another occasion.

Research on $\mathrm{CO}$ has been partly driven by observations of the strikingly deep cores of the strongest lines, which are still not fully explained (see Tsuji 1988, 1991; Judge 1989). Simply stated, certain CO lines in the fundamental band are so strong their cores must be formed in the same part of the atmosphere as the $\mathrm{Ca}$ II $\mathrm{H}$ and $\mathrm{K}$ lines or even the $\mathrm{Mg}$ II $\mathrm{h}$ and $\mathrm{k}$ lines. Both of these latter sets of lines show emission features reflecting the chromospheric temperature rise. Theory suggests these CO lines are formed by pure absorption (LTE), and, if so, they must also develop emission cores (Carbon et al. 1976; Ayres \& Wiedemann 1989; Wiedemann \& Ayres 1991). However, no emission features are observed in spectra of any star (Heasley et al. 1978; Tsuji 1988), including such diverse objects as Sun, Arcturus, and TX Psc (a carbon star). How to understand this conflict? Either CO must be subject to departures from LTE so that the radiation temperatures in its line cores do not correspond to electron temperatures in the stars, or the chromospheres must be inhomogeneous, with cool regions (where $\mathrm{CO}$ is formed) and warmer regions (where the resonance lines of $\mathrm{Ca}$ II and $\mathrm{Mg}$ II are formed). In fact, this latter point of view has been expressed by several authors, but the matter appears to be far from settled.

Pioneering work (Thompson 1973) based upon available cross sections showed that (a) rotational equilibrium must hold throughout the atmospheres of almost all stars, (b) vibrational equilibrium might break down in the the low-density atmospheres of the coolest giants, and (c) ionization and chemical equilibria ought to be in LTE. A later comprehensive overview of line formation in several molecules (Hinkle \& Lambert 1975) again considered rotational, vibrational, and electronic transitions and attempted to distinguish observationally between lines formed by pure absorption and scattering (favoring the latter), disclosed the uncertainties of the cross sections used earlier, and emphasized the importance of collisions with neutral hydrogen for ground-state rovibrational transitions.

A calculation of NLTE in CO in Arcturus through the solution of the equations of radiative transfer and statistical equilibrium (Carbon et al. 1976) illustrated how sensitively the results depended upon the poorly known cross sections for $\mathrm{H}-\mathrm{CO}$ collisions. They delineated the regimes of temperature and pressure in which departures from LTE might be expected in rovibrational transitions, and found that LTE would hold (for the "best" cross sections) throughout the atmosphere except perhaps the outermost layers. In addition, these authors pointed out that the $\mathrm{CO}$ results could not be reconciled with a chromospheric model and pointed toward an inhomogeneous outer atmosphere.

To address the problem of NLTE in CO directly, Ayres \& Wiedemann (1989) undertook a careful NLTE calculation of the rotation-vibration lines in the Sun. A NLTE calculation for a molecule is a formidable problem. Consider a molecule 
of 10 vibrational levels, each with 121 rotational levels. Imagine well over 1,000 radiative transitions to be accounted for. That is the molecular model adopted. Fortunately, because the collisions are dominated by neutral hydrogen (for which reliable cross sections were then known), the value of the fractional absorption parameter, $\epsilon$, essentially the rate of collisional to radiative de-excitation, is fairly large, of the order of 0.01 to 0.10 . The solution of the radiative transfer equation can therefore be found by straightforward lambda iteration, and the solution thermalizes at relatively shallow depths. They found that the deep cores seen in strong infrared $\mathrm{CO}$ lines in Sun and Arcturus could not be explained by NLTE. This interesting result, that LTE holds for infrared CO lines, must not be confused with the fact of fluorescence, a selective NLTE effect, seen in some ultraviolet CO lines (Ayres 1986).

The NLTE research on the rotation-vibration transitions and levels within the ground electronic state $\left(\mathrm{X}^{1} \Sigma^{+}\right)$in $\mathrm{CO}$ was later extended to $\mathrm{F}-\mathrm{K}$ stars (Wiedemann \& Ayres 1991) based on standard radiative- equilibrium, LTE models (Gustafsson et al. 1975; Bell et al. 1976), where again only slight departures from LTE were found in atmospheres for stars with $\log$ (surface gravity) $\geq 1.5$, which includes all dwarfs and most giants. Model makers had previously employed the LTE assumption, and it has been used in all later work on CO opacities (Plez et al. 1992), line formation (Jørgensen \& Johnson 1992), and cooling calculations (Mauas et al. 1990).

As part of a novel calculation of a self-consistent model for the solar photosphere under the constraints of hydrostatic and radiative equilibrium but without the LTE constraint (Anderson 1989), calculations were carried out for several molecules, including the $\mathrm{CO}$ fourth positive system (A-X). Interesting departures from LTE were found in the sense that in the outer solar atmosphere (outside the temperature minimum in a real red-giant atmosphere) all levels were overexcited; that is, all levels were overpopulated relative to LTE. The cooling effects of CO were confirmed (Anderson 1989), but the effects are much more complex than previously treated because of the heating and cooling effects of strong atomic lines. Questions of bifurcation are outside our considerations here.

\section{$6 \mathrm{TiO}$}

Following $\mathrm{H}_{2} \mathrm{O}$, TiO is the dominant molecular opacity in oxygen-rich atmospheres, and the red and infrared spectral regions are blanketed by millions of lines of several systems: $\alpha\left(\mathrm{C}^{3} \Delta-\mathrm{X}^{3} \Delta\right), \beta\left(\mathrm{c}^{1} \Phi-\mathrm{a}^{1} \Delta\right), \gamma^{\prime}\left(\mathrm{B}^{3} \Pi-\mathrm{X}^{3} \Delta\right), \gamma$ $\left(\mathrm{A}^{3} \Phi-\mathrm{X}^{3} \Delta\right), \delta\left(\mathrm{b}^{1} \Pi-\mathrm{a}^{1} \Delta\right), \phi\left(\mathrm{b}^{1} \Pi-\mathrm{d}^{1} \Sigma\right), \epsilon\left(\mathrm{E}^{3} \Pi-\mathrm{X}^{3} \Delta\right)$. Some TiO opacities have been employed by all constructors of cool-star atmospheres (Brown et al. 1989; Bessell et al. 1989; Plez et al. 1992; Jørgensen 1993). As far as we know, no NLTE analysis has been carried out for TiO in any star. Comments regarding possible NLTE effects on TiO are made in Section 8. 


\section{Polyatomic Molecules}

Even though polyatomic molecules provide very important opacities $-\mathrm{H}_{2} \mathrm{O}$ in oxygen rich stars and $\mathrm{HCN}, \mathrm{C}_{2} \mathrm{H}_{2}$, and $\mathrm{C}_{3}$ in carbon stars- we know of no research on NLTE in these molecules. We note that more complex molecules have more pathways for any given transition $i \leftrightarrow j$, which increases opportunities for thermalization (LTE). Furthermore, all the important spectral bands of the molecules (except perhaps the $\mathrm{A}-\mathrm{X}$ transition of $\mathrm{C}_{3}$ ) are due to transitions within the electronic ground state (infrared rovibrational transitions), and are therefore less affected by NLTE as described in Section 1. For the near future, we expect all calculations of dissociative equilibrium among polyatomic molecules to continue to use LTE. Formation and destruction of PAHs (polycyclic aromatic hydrocarbons), polyynes, fullerenes, and other complex molecules are discussed by Omont (this volume).

\section{NLTE in the Pool of Atoms}

While scientists have worried about possible departures from LTE in stellar atmospheres, because almost all calculations to date have used this marvelously simplifying assumption, effects of NLTE in photospheres where molecules exist have not been easy to find. In this section we are concerned whether ionization equilibrium exists among molecule-forming atoms since such departures would change the pool of neutral atoms from which molecules might form. A few examples must suffice.

Most diatomic molecules are composed of $\mathrm{H}, \mathrm{C}, \mathrm{N}$, and $\mathrm{O}$, which share the trait of sufficiently high ionization energy $(11.3-14.5 \mathrm{eV})$ that they are almost entirely neutral in all stars cooler than the Sun. Even though NLTE effects (overionization) are present (Ayres \& Weidemann 1989; Anderson 1989; Luttermoser \& Johnson 1992; Luttermoser 1993) and the number of ions is therefore greatly increased over LTE calculations, the enormously greater number of neutral atoms is not significantly diminished. Thus, the number density of molecules composed of these abundant atoms is not affected by NLTE.

A pioneering calculation of ionization equilibria in cool giant stars was carried out by Auman \& Woodrow (1975) for the elements $\mathrm{Na}, \mathrm{Al}, \mathrm{K}$, and $\mathrm{Ca}$ in several model photospheres for red-giant stars ranging from 2,000 to $4,000 \mathrm{~K}$ in effective temperature. Each element was represented by a model atom with 9 energy levels. Atmospheric models based on LTE were used as starting values, ultraviolet radiation fields were calculated from the models, and the radiative-transfer and steady-state equations were solved self-consistently. These results were used to obtain new models, and the process was iterated to convergence to create NLTE model atmospheres. In the outer atmospheric layers overionization was found for all elements considered, and this overionization increased with decreasing temperature and gravity up to a factor of about 10 for $T_{\text {eff }}=3500 \mathrm{~K}$, for example.

A dependence of the (LTE) abundance of Fe I and $\mathrm{Ti}$ I on the excitation potential as well as the equivalent width of spectral lines in the star Pollux (K0 
III) has been put forward as evidence of departures from LTE in the photosphere (Ruland et al. 1980). A similar effect in $\mathrm{Ti} \mathrm{I}$ and $\mathrm{Zr} \mathrm{I}$ was noticed in a study of $33 \mathrm{G}$ and $\mathrm{K}$ giants by Brown et al. (1983), who found that the $\mathrm{Zr}$ abundance was 0.4 dex lower, relative to $\mathrm{Ti}$, than in the Sun when weak $\mathrm{Zr}$ I and $\mathrm{Ti}$ I lines were used for the abundance determination. However, these "abundance" effects disappeared when lines of $\mathrm{Ti}$ II and $\mathrm{Zr}$ II were employed, indicating that NLTE effects were responsible. These authors warn other researchers to use only the most abundant species of an element for abundance determinations.

A landmark study of complex atoms in stars was the pioneering research on line formation of $\mathrm{Fe}$ in the solar atmosphere (Athay \& Lites 1972), who carried out a solution of the coupled steady-state and radiative transfer equations for a model atom of 15 terms and 13 radiative bound-bound transitions. This work was later extended to similar stars (Lites \& Cowley 1974) with somewhat simpler ( $\mathrm{Fe} \mathrm{I}+\mathrm{Fe}$ II atoms (12 atomic levels and 5 radiative bound-bound transitions).

Theoretical calculations for more complex model atoms (69 spectroscopic terms and 67 transitions) of Fe I and Fe II (Steenbok 1985) in the Sun and in Pollux have largely explained theoretically the results obtained by Ruland et al. (1980), thus putting the research on NLTE in complex atoms on a rather more solid footing. This same work was later elaborated further with an even more complex atom (Solanki \& Steenbok 1988), but even this model atom was a rather crude approximation to the real iron atoms.

A novel calculation of a theoretical solar atmospheric model based upon radiative and hydrostatic equilibrium but without the LTE assumption has been made (Anderson 1989). In this calculation, the equations of statistical equilibrium (similar to Eq. 5) and radiative transfer (Eq. 2) were solved self-consistently for those atoms and molecules which account for most of the line blanketing: model neutral atoms of $\mathrm{H}, \mathrm{He}, \mathrm{C}, \mathrm{Na}, \mathrm{Mg}, \mathrm{Al}, \mathrm{Si}, \mathrm{Ca}$, and $\mathrm{Fe}$ and model molecules for $\mathrm{CH}, \mathrm{CN}, \mathrm{CO}$, and $\mathrm{SiO}$.

This is a numerically difficult and computationally expensive undertaking, and several simplifications were necessary. In particular, the "multifrequency/multigray" approximation was employed for the opacity, which effectively reduces a complex atom or molecule to a set of representative multiplets for which moreor-less exact solutions can be obtained, and from which extrapolations to the complete solution can be made. Molecular dissociative equilibrium was treated in LTE. Although the abundance of their ions was increased relative to LTE, molecule-forming atoms remained the dominant stage of ionization, and molecular formation was therefore treated self-consistently in LTE.

In the outer photospheric layers the temperature in a NLTE model falls far below the temperature of an LTE model atmosphere. Molecular lines, treated by the complete-redistribution (CRD) formulation (Eqs. 11 and 12), departed from the predictions of pure absorption, and this resulted in interesting effects on (low-frequency) cooling and (high-frequency) heating. Again it was found that molecular energy levels are overexcited, sometimes by a large factor, in the outer atmosphere (above $\log m=2.0$ ).

More recently, Takeda (1991) has noted the deficiencies in the atomic models 
of iron adopted by previous investigators because of the limited number of levels and transitions considered and has solved self-consistently the radiative-transfer and steady-state equations for a model $\mathrm{Fe} I$ atom of sufficiently many levels and lines to include the effects of huge numbers (hundreds) of highly excited states and weak lines. These were, of course, treated by a statistical procedure, in which an actual multiplet or term is represented by a single fictitious level with an appropriate transition probability/statistical weight in a manner similar to that of Anderson (1989). The model atmosphere was chosen to represent Arcturus (K2 III). Because the photon re-emission mechanism could not be known for all lines, calculations on opacities in the ultraviolet were made for both pure absorption and for pure coherent scattering. Although the NLTE factors differ by a small factor $(\sim 1-3)$ depending on the inclusion or exclusion of scattering, the results differ by an order of magnitude if line-blanketing is included (as it should be) in the ultraviolet. In other words, the extent of Fe I overionization is mostly fixed by the treatment of ultraviolet fluxes and therefore line opacities. The results show a considerable overionization of $\mathrm{Fe} I$ in the outer atmosphere, above $\log \tau(500 \mathrm{~nm})=-3.0(\log$ column mass density $\sim 1.0)$. Cores of strong and intermediate lines are formed above this region, and these results suggest a possibly significant overionization of Fe I.

If this result for $\mathrm{Fe}$ can be extrapolated to $\mathrm{Ti}$, it might prove very interesting for the formation of $\mathrm{TiO}$ lines. Similarly, a NLTE calculation for $\mathrm{Mg}$ would test NLTE effects on MgH.

\section{Conclusions and Discussion}

Although NLTE effects in molecular opacities might arise in several contexts, the problems of calculating these are complex and poorly studied. We make the following comments.

1. Rotational levels, which have energy differences of $0.00-0.05 \mathrm{eV}$ and whose lines fall in the red and infrared region, are in LTE because of the high collisional rate (due to neutral hydrogen) relative to the modest radiative rates.

2. Vibrational levels, with energy differences of $0.1-0.5 \mathrm{eV}$, are also likely to be in LTE for the reasons given above although departures may occur in some molecules in the outermost layers of cool supergiants or miras. These transitions are responsible for much of the opacity in cool stars.

3. Molecular electronic transitions, like atomic transitions, require energies of several $\mathrm{eV}$. Collisions in these transitions depend on electrons, which are relatively rare in these cool objects, where abundant elements are generally neutral. Significant departures in populations of energy states may result, although no NLTE effects have been found observationally. Bands from electronic transitions in many molecules fall in the violet and ultraviolet spectral regions where their opacities are not significant (but note such exceptions as $\mathrm{CN}$ ) for the thermal structure (though they are significant for violet and ultraviolet spectral synthesis) even in the Sun. They rapidly become less important in cooler stars. 
4. We speculate that the most significant effect of NLTE on molecular opacities will arise from overionization of neutral atoms, which might reduce the pool from which molecules can be made. This effect will not occur for molecules composed of the abundant elements $\mathrm{H}, \mathrm{C}, \mathrm{N}$, and $\mathrm{O}$ because their large (11-15 eV) ionization energies assure they will be predominantly neutral throughout coolstar atmospheres. We suggest NLTE effects might arise in such an important opacity as $\mathrm{TiO}$ if $\mathrm{Ti} \mathrm{I}\left(\chi_{i o n}=6.8 \mathrm{eV}\right)$ is actually overionized when it would have been neutral in LTE. Although not a significant opacity source, $\mathrm{MgH}$, which is valuable for pressure and gravity determinations, might be affected in the same way if $\mathrm{MgI}\left(\chi_{i o n}=7.6 \mathrm{eV}\right)$ is overionized.

5. LTE will continue to be employed for the foreseeable future in most calculations of molecular opacities, model atmospheres, and elemental and isotopic abundances. Research in the outer photosphere, chromosphere, and CSE will require NLTE. The exact region of overlap is a subject of current research.

Acknowledgement. The author expresses appreciation to the Organizing Committee of Colloquium 146 and to Indiana University for travel grants. Helpful discussions with Lawrence Anderson and Tom Ayres are acknowledged.

\section{References}

Anderson L.S., 1989, Astrophys. J.,339, 558

Athay R.G., Lites B.W., 1972, Astrophys. J., 176, 809

Auman J.R., Woodrow J.E.J., 1975, Astrophys. J., 197, 163

Ayres T.R., 1986, Astrophys. J., 308, 246

Ayres T.R., Wiedemann G.R. 1989, Astrophys. J., 338, 1033

Bell R.A., Eriksson K., Gustafsson B., Nordlund $\AA$, 1976, Astron. Astrophys. Suppl., 23, 37

Bessell M.S., Brett J., Scholz M., Wood P.R., 1989, Astron. Astrophys. Suppl., 77, 1

Bowen G.H., 1988, Astrophys. J., 329, 299

Brown J.A., Johnson H.R., Alexander D.R., Cutright L.C., Sharp C., 1989, Astrophys. J. Suppl., 71, 623

Brown J.A., Tomkin J., Lambert D.L., 1983, Astrophys. J., 265, L93

Carbon D.F., Milkey R.W., Heasley J.N., 1976, Astrophys. J., 207, 253

Carpenter K.G., Robinson R.B., Wahlgren G.M., Linsky J.L., Brown A., 1993, Astro* phys. J., submitted

Grevesse N., Lambert D.L., Sauval A.J., van Dishoeck E.F., Farmer C.B., Norton R.H., 1991, Astron. Astrophys., 242, 488

Gustafsson B., Bell R.A., Eriksson K., Nordlund $\AA$, 1975, Astron. Astrophys., 42, 407

Heasley J.N., Ridgway S.T., Carbon D.F., Milkey R.W., Hall D.N.B., 1978, Astrophys. J., 219, 790

Hinkle K.H., Lambert D.L., 1975,it MNRAS, 170, 447

Hinkle K.H., Martin C.L., Ridgway S.T., 1993, Astrophys. J., in press

Johnson H.R., 1973, Astrophys. J., 180, 81

Jørgensen U.G., 1993, Astron. Astrophys., in press

Jørgensen U., Johnson H.R., 1991, Astron. Astrophys. 244, 462

Jørgensen U.G., Johnson H.R., Nordlund $\AA$., 1992, Astron. Astrophys.,261, 263 
Judge P.G. 1989, Evolution of Peculiar Red Giant Stars, ed. H.R. Johnson \& B. Zuckerman, Cambridge Press, Cambridge, 303

Lambert D.L., Pagel B.E.J., 1968,MNRAS, 141, 299

Lites B.W., Cowley C.R., 1974, Astron. Astrophys.,31, 361

Lites B.W., Mihalas D., 1984, Solar Phys., 93, 23

Luttermoser D.G. 1993, Astrophys. J., in preparation

Luttermoser D.G., Johnson H.R. 1992, Astrophys. J.,388, 579

Mauas P.J., Avrett E.H., Loeser R. 1990, Astrophys. J., 357, 279

Mihalas D., 1978, Stellar Atmospheres (2nd ed.), Freeman, San Francisco, chap.12

Mount G.H., Ayres T.R., Linsky J.L., 1975, Astrophys. J., 200, 383

Mount G.H., Linsky J.L., 1975,Solar Physics, 41, 17

Plez B., Brett J.M., Nordlund $\AA$., 1992, Astron. Astrophys., 256, 551

Ruland F., Holweger H., Griffin R., Griffin R., Biehl D., 1980, Astron. Astrophys., 92, 70

Schrijver C.J., 1987, Astron. Astrophys., 172, 111

Solanki S.K., Steenbok W., 1988, Astron. Astrophys., 189, 243

Steenbok W., 1985,in Cool Stars with Excesses of Heavy Elements, ed. M. Jaschek, P.C. Keenan, Reidel, Dordrecht, 231

Steiman-Cameron T.Y., Johnson H.R., Honeycutt R.K., 1985, Astrophys. J., 291, L51

Takeda Y., 1991, Astron. Astrophys., 242, 455

Thompson R.I., 1973, Astrophys. J., 181, 1039

Tsuji T., 1986, Ann. Rev. Astron. Astrophys., 24, 89

Tsuji T., 1988, Astron. Astrophys.,197, 185

Tsuji T., 1991, Astron. Astrophys., 245, 203

Vernazza J.E., Avrett E.H., Loeser R., 1981, Astrophys. J. Suppl., 45, 635

Wiedemann G., Ayres T.R. 1991, Astrophys. J., 366, 277 